\title{
A model metallic glass exhibits size-independent tensile ductility
}

\author{
Jian Luo, Pawel Keblinski, and Yunfeng Shi \\ Department of Materials Science and Engineering, \\ Rensselaer Polytechnic Institute, Troy, NY 12180
}

\begin{abstract}
Metallic glasses (MGs) usually suffer brittle fracture under uniaxial tension due to size-dependent shear band cavitation. Here we developed a concurrent multi-scale simulation method to describe the uniaxial tension of a binary Lennard-Jones (BLJ) model glass up to 88 microns in length. No cavitation or brittle fracture was found even for the longest BLJ sample. As the length increases, the shear band temperature increases, then saturates, while the elastic unloading from shear-off diminishes. We conjecture that the shear band of a BLJ sample, even with a macroscopic length, cannot reach the herein estimated critical cavitation conditions. Thus, BLJ samples appear to be free of size-induced tensile brittleness. Based on the shear band evolution and the critical cavitation conditions, we propose three classes of MGs in terms of tensile ductility: brittle-MGs (brittle for all lengths), normal-MGs (ductile for short samples, brittle for long samples), cohesive-MGs (ductile for all lengths). Our simulation results illustrate limitations of existing molecular models, and suggest that certain experimental metallic glasses may be free of size-induced tensile brittleness.
\end{abstract}

(C) 2015. This manuscript version is made available under the Elsevier user license http://www.elsevier.com/open-access/userlicense/1.0/ 


\section{Introduction}

Metallic glasses (MGs) can exhibit high toughness[1], high strength[2-4] and excellent formability[5,6]. Although certain amount of ductility can be achieved under both compression and bending[2,3,7,8], monolithic metallic glasses lack tensile ductility, constituting the major drawback for load bearing applications. To achieve tensile ductility, various microstructural engineering approaches have been investigated, including the introduction of crystalline phases[9,10], regular pores[11], notches[12,13], or structural gradient[14]. Aside from microstructural engineering, it is generally believed that the tensile brittleness of metallic glasses is size-dependent, which has been demonstrated experimentally[4,15-17]. Thus, an alternative approach to achieve tensile ductility is to reduce the MG sample length.

However, the size-dependent tensile ductility in MG system has not been fully understood[4,16-23]. The brittle tensile fracture of macroscopic MG sample generally results from the development of a run-away shear band, which transits to a crack via shear band cavitation, causing catastrophic failure. The critical shear band cavitation process occurs within nano-second in a shear band of nano-meter thickness, on which direct quantitative experimental characterization is extremely challenging[24]. To this end, atomic-scale simulations[25-29] with high temporal and spatial resolution become increasingly important to understand the fracture process of metallic glasses. However, there are only few atomic-scale simulations on size-dependency of MG systems. We have previously shown ductile-to-brittle transition as sample length increases in a model modified binary Lennard-Jones (mBLJ) system using full-atomic molecular dynamics (MD) simulations[30]. To our best knowledge, there is no report on other model glass forming system that exhibits such size-dependent behavior in uniaxial tension. One possibility is that the critical length for the ductile-to-brittle transition is too long to simulate with full MD simulations. Alternatively, such size-dependent tensile ductility may not be universal among all glass formers.

To address this issue, we selected a well-studied Wahnstrom binary Lennard-Jones (BLJ) system to examine whether it exhibits size-dependent tensile 
ductility. To simulate long samples, we designed a concurrent multi-scale simulation technique to model uniaxial tension test on metallic glass samples with a length up to 88 microns. The multi-scale scheme combines an atomic region containing the shear band and the vicinity, while the rest of the sample is modeled at the continuum level. This scheme takes advantage of the fact that plasticity and possible cavitation only occur in a highly localized region, i.e., the shear band, while the rest of the material serves merely as heat-conducting elastic medium. Based on the estimated critical shear band cavitation conditions and the thermomechanical evolution of the shear band under uniaxial tension, we conjecture that BLJ system does not exhibit shear band cavitation at infinite length. Therefore, BLJ system does not exhibit size-dependent tensile ductility, instead, exhibits size-independent ductility under uniaxial tension. We further propose a classification of metallic glasses in terms of the tensile ductility.

\section{Multi-scale simulation setup}

The brittle fracture of a typical MG sample under uniaxial tension proceeds as follows. The sample first elastically deforms, usually followed by the development of a dominant shear band. The shear band then glides, and may transit to crack via cavitation within the shear band. Therefore, the shear banding region (typically nm-in-thickness) plastically flows and cavitates, while the rest of sample only deforms elastically and conducts heat, as shown in Fig. 1. Such situation is well suited for a concurrent multi-scale simulation: the atomic-level details in the shear band and its vicinity (very small region of the sample, shown in the right pane of Fig. 1) will be captured by MD simulations; the elastic response and thermal conduction of the rest of the sample will be described at the continuum-level. Note that periodic boundary conditions are present in all three directions in the MD region. This multi-scale simulation strategy allows significant speed-up compared to the full-MD simulations, thus permitting simulations of samples up to 88 microns. 
Similar to our previous work on shear band cavitation[30], we considered a thin-slab sample under plane strain conditions (corresponding to a thick sample in experiments). The mechanical and thermal coupling between the MD simulation and the continuum-level modeling will be described in details below.

\subsection{Mechanical coupling}

Elastic regime. First we will establish the relations for both stress and strain between the MD region and the entire sample. As shown in Fig. 1, the stresses in the MD region (the shear band and its vicinity) are denoted as $\sigma_{x x}, \sigma_{z z}$ and $\sigma_{z X}$, in the Cartesian system of xyz. $x-, y-, z-$ axes point along the shear band direction, the paper normal direction (or the thickness direction of the slab), and the direction normal to the shear band, respectively. The tensile stress of the entire sample is denoted as $\sigma_{L}$. Resolving the tensile stress of the sample, it is straightforward to have,

$$
\begin{aligned}
& \sigma_{x x}=\sigma_{L} \cos ^{2} \theta \\
& \sigma_{z x}=\sigma_{L} \sin \theta \cos \theta \\
& \sigma_{z z}=\sigma_{L} \sin ^{2} \theta
\end{aligned}
$$

$\theta$ is the angle between the soon-to-be-formed shear band and the loading direction. Here $\theta$ is taken as 45 degrees. Similarly, one can also relate the loading strain $\varepsilon_{L}$ of the whole sample to the tensile strain $\varepsilon_{z}$ of the MD region. As shown in Fig. 1, the horizontal contraction strain of the whole sample is $\frac{v}{1-v} \varepsilon_{L}$ given plane strain conditions ( $v$ is the Poisson's ratio). Through strain tensor rotation, one can obtain,

$$
\varepsilon_{z z}=\left(\sin ^{2} \theta-\frac{v}{1-v} \cos ^{2} \theta\right) \varepsilon_{L}
$$

The elastic deformation of the continuum regime is trivial as the elastic constants of the model MG system are known. Nonetheless, one needs to elastically load the $\mathrm{MD}$ region that is consistent with the shear band angle. Here, the MD region is 
subjected to a constant strain rate tension in the direction normal to the shear band (zdirection, as shown in Fig. 1), with two barostats that control the normal stresses in the transverse direction $\left(\sigma_{x x}\right)$ and the shear stress $\left(\sigma_{x z}\right)$ as follows,

$$
\begin{aligned}
& \dot{\varepsilon}_{z z}=\left(\sin ^{2} \theta-\frac{v}{1-v} \cos ^{2} \theta\right) \dot{\varepsilon}_{L} \\
& \sigma_{x x}=\sigma_{z z} \cot ^{2} \theta \\
& \sigma_{z x}=\sigma_{z z} \cot \theta
\end{aligned}
$$

Here $\sigma_{z z}$ is the instantaneously measured normal stress in the z-direction. In this way, within the elastic loading regime, mechanical equilibrium between the continuum regime and the MD regime (i.e., Eqs. 1-3) is guaranteed. Furthermore, the stress-strain response of the whole sample can be obtained from the MD simulation: the tensile stress of the whole sample $\sigma_{L}$ can be obtained according to Eq. 3 from $\sigma_{z z}$, and the strain of the whole sample $\varepsilon_{L}$ can be obtained according to Eq. 4 from $\varepsilon_{Z Z}$

Plastic regime. During plastic deformation, mechanical equilibrium, guaranteed in the elastic regime, can be violated due to localized shear banding. Fig. 2 shows the driving resolved shear stress $\sigma_{z z} \cot \theta$ and the resisting shear stress $\sigma_{z x}$ using the elastic coupling scheme described above. It is apparent that as shear band forms (the visible drops in both stresses due to structural softening[25,31]), mechanical equilibrium can no longer be maintained. Here we identify the system configuration just prior to shear band formation, as $\sigma_{z x}$ becomes lower than $\sigma_{z z} \cot \theta$ (occurs around $5.5 \%$ in Fig. 2), from which a new mechanical coupling scheme proper to plastic deformation will be employed.

The central physical process during plastic deformation regime is the shear band gliding, which is described both in the MD region (as characterized by the shear strain $\varepsilon_{z X}$ ) and the continuum region (as characterized by the shear offset $S_{g}$ ). Thus, one can 
link the shear strain in MD region to the shear offset in the continuum region. Another mechanical coupling arises from the assumption that the mechanical equilibrium conditions are maintained along the shear band ( $x$ - direction) and normal to the shear band ( $z$ - direction). This is because there is no apparent dialatation/contraction in the $x$ - direction, and negligible dilatation in the $z$ - direction until cavitation. Therefore, Eqs. (1 and 3) are still valid in the plastic deformation regime. We will describe below how the continuum description informs MD, and then how the MD simulation provides input for the continuum calculations.

The MD system during plastic deformation is subjected to a strain-controlled shear deformation with a shear strain rate of $\dot{\varepsilon}_{x z}$ with $x$ - and $z$ - direction coupled to two stress-controlled barostats. The third dimension of the system is fixed, in the spirit of plane strain conditions. From the continuum descriptions, the shear strain rate relates to the velocity of the shear offset $\dot{S}_{g}(t)$, while the two normal stresses are determined by the tensile stress $\sigma_{L}$,

$$
\begin{aligned}
& \dot{\varepsilon}_{x z}=\frac{\dot{S}_{g}(t)}{l_{z}} \\
& \sigma_{z z}=\sigma_{L} \sin ^{2} \theta \\
& \sigma_{x x}=\sigma_{L} \cos ^{2} \theta
\end{aligned}
$$

Here $\dot{S}_{g}(t)$ is the speed of shear offset, while $l_{z}$ is the MD simulation box size in the $z$-direction. For convenience, the beginning of the plastic regime is time zero $(t=0)$.

For the continuum side, we consider the sample with two equal parts (connected at the shear band) that can move relative to each other and elastically deform, as shown in Fig. 1. Let us first consider their accelerations and resulting shear offset. Once shear band forms, mechanical equilibrium is broken which leads to acceleration of the sample. Here, it is assumed that the machine stiffness is infinite without any tangential force from the grips to the sample (free to move tangentially to the loading direction), in order to be compatible with our earlier study[30]. For either the top or bottom half of the sample, the total force experienced along the shear band direction 
is $\left(\sigma_{z z}(t) \cot \theta-\sigma_{z x}(t)\right) \frac{A}{\sin \theta}$, where $A$ is the cross-section area of the sample. The component of this force along the loading direction is balanced by the grips, while the component of this force along the direction perpendicular to the tensile loading is unbalanced as the grips are free to slide tangentially. Therefore, the acceleration along the direction normal to the loading direction (i.e., the horizontal direction in Fig. 1) is,

$$
a(t)=\frac{\left(\sigma_{z z}(t) \cot \theta-\sigma_{z x}(t)\right) \frac{A}{\sin \theta} \sin \theta}{\rho L A / 2}
$$

Here, $a, L$, and $\rho$ are the acceleration, the length of the sample, and the density, respectively. As there is no horizontal motion at time zero, the net horizontal displacement can be written as $\int_{0}^{t} \int_{0}^{\tau} a(\xi) d \xi d \tau$. Thus, the horizontal offset between the top and bottom half of the sample becomes $2 \int_{0}^{t} \int_{0}^{\tau} a(\xi) d \xi d \tau$. Given that the sample does not fracture, from pure geometrical consideration, the shear offset along the shear band direction is $\frac{2}{\sin \theta} \int_{0}^{t} \int_{0}^{\tau} a(\xi) d \xi d \tau$. Thus, given zero shear offset and zero gliding speed at time zero (beginning of the plastic regime), we have,

$$
S_{g}(t)=\frac{4}{\rho L \sin \theta} \int_{0}^{t} \int_{0}^{\tau}\left(\sigma_{z z}(\xi) \cot \theta-\sigma_{z x}(\xi)\right) d \xi d \tau
$$

and its time derivative,

$$
\dot{S}_{g}(t)=\frac{4}{\rho L \sin \theta} \int_{0}^{t}\left(\sigma_{z z}(\tau) \cot \theta-\sigma_{z x}(\tau)\right) d \tau
$$

Once the shear offset is known, the instantaneous tensile stress of the sample can be written considering the sample elastically unloading (second term of the RHS of Eq. 14) and constant-strain-rate loading from the grips (third term of the RHS of Eq. 14)[21],

$$
\sigma_{L}(t)=\sigma_{L}(0)-\frac{S_{g}(\mathrm{t}) \cos \theta}{L} E+\dot{\varepsilon}_{L} t E
$$


Here $\sigma_{L}(t), \sigma_{L}(0), E, \dot{\varepsilon}_{L}$, and $t$ are the instantaneous tensile stress of the entire sample, the initial tensile stress at the beginning of the plastic regime, the Young's modulus, the tensile strain rate of the entire sample, and time, respectively. Here we consider the ideal situation that the machine stiffness is infinite and machine inertia is zero[21]. It is assumed that mechanical equilibrium establishes instantaneously within the continuum region, thus ignoring elastic wave propagation observed in full atomic simulations[30]. Eq. (12-14) provide the continuum descriptions of shear offset and tensile stress of the sample during shear band sliding using MD simulation data (the instantaneous stresses) as inputs.

\subsection{Thermal coupling}

The thermal coupling in the elastic loading regime is uniform, thus thermal coupling is trivial. However, in the plastic regime, the shear band gliding leads to significant local heat generation. Subsequently, heat flows out of the MD region to the continuum region. Therefore, it is essential to implement the thermal coupling between the MD region and the continuum region in the plastic regime.

For the continuum description of the temperature evolution, we simplify it into a 1D thermal diffusion problem in an infinitely long grid, along the direction perpendicular to the shear band (z-direction). Thus, the temperature variations parallel to the shear band ( $x$-direction) or through the thickness of the sample ( $y$-direction) are omitted. The temperature grid has a grid size of $0.9 \mathrm{~nm}$. The grid will automatically extend once the heat front reaches a given region. The heat front is identified once the temperature rise of the end grids exceeds $0.17 \mathrm{~K}$.

We divided the system along the z-direction into three types of regions as shown in Fig. 3. The MD system spans over both Type I and Type II regions, while the continuum description exists over the entire Z-direction including Type I, II and III. The MD system within Type I region evolves without coupling to any thermostat. The MD system within Type II regions are coupled to location-specific thermostats matching to the continuum grids, with the grid temperatures as targets. The continuum 
model within Type I region is taken solely from coarse-graining the MD simulation results. The continuum model within Type II and III regions evolve according to Fick's law,

$\frac{\partial T}{\partial t}=\frac{\kappa}{\rho c_{p}} \frac{\partial^{2} T}{\partial z^{2}}$

$\kappa$ is the thermal conductivity, measured via the Muller-Plathe algorithm[32] at different temperatures, which increases from 0.75 to about $1.05 \mathrm{~W} / \mathrm{K} / \mathrm{m}$ from $10 \mathrm{~K}$ to $1000 \mathrm{~K} . c_{p}$ is the specific heat capacity, which stays constant until $\mathrm{T}$ approaches $\mathrm{T}_{\mathrm{g}}$ around $1000 \mathrm{~K} . \rho$ is the density. Since the temperature in Type II and III regions is generally lower than $\mathrm{T}_{\mathrm{g}}, c_{p}$ and $\rho$ are assumed to be constants in the continuum model. The temperature gradient in Eq. (15) is calculated numerically using the second order central difference approximation. The continuum model is updated every $0.05 \mathrm{ps}$ (which is $10 \mathrm{MD}$ timesteps).

The initial temperature values of the continuum grids at time zero is uniform at $\mathrm{T}=10 \mathrm{~K}$. The thermal coupling between the MD simulations and the continuum modeling can be described as follows. Between two updates of the continuum grids, the continuum grids provide the boundary conditions for the MD simulations: the temperature distribution in Type II region of the continuum region sets the thermostats in Type II region of the MD simulation which allows heat flowing out. During the update of the continuum grid, the MD simulation results provide the boundary conditions for the continuum grids: the temperature sets by the MD simulations in Type I region heats up the Type II region of the continuum grids.

It should be noted that, between two updates of the continuum grids, the two Type II regions of the MD system is in thermal contact due to the periodic boundary conditions. To minimize such unphysical heat exchange, once the shear band forms in the MD region, the system is translated along the $z$-direction such that the shear band locates at the center of the simulation box. In this way, the temperature distribution will be approximately mirror-symmetric for those two Type II regions to avoid heat exchange. 


\subsection{Verification of the multi-scale simulation method}

Here the multi-scale simulation method was tested against the full MD simulation in terms of uniaxial tensile tests. The size of the sample is relatively small $\left(22.5 \times 2.5 \times 45 \mathrm{~nm}^{3}\right)$ to allow full MD simulation. In the multi-scale method, the size of the atomic region is $7.5 \times 2.5 \times 15 \mathrm{~nm}^{3}$. Three independent multi-scale simulation were performed to examine the statistic variation.

First, the gliding distance $S_{g}$ of the shear band from both the full MD simulation and the three independent multi-scale simulations were shown in Fig. 4 (a). Both the initial "burst" of shear band gliding at about 5.5\% strain, and the later "steady-state" gliding are consistent between these two methods. In addition, as shown in Fig. 4(b), the stress-strain curves from the three independent multi-scale simulations match relatively well to the stress-strain curve of the direct MD simulation. It should be noted that the yield stress in full MD simulation is slight lower than that of multi-scale simulation, which is likely due to the surface effect. Moreover, the stress during plastic flow in full-MD simulation is higher than the multi-scale simulations. The shear band in multi-scale simulation is perfectly straight, which is more efficient in unloading. Finally, the temperature distribution evolution in the multi-scale simulations all converge to that of the direct MD simulation at large strains. Thus the multi-scale simulation method can reasonably reproduce the thermomechanical response of the glassy sample, with a dominant shear band, during uniaxial tension tests.

Full atomic simulations on the modified BLJ samples have shown size dependent tensile fracture via shear band cavitation[30]. Here, we employ the multi-scale simulation to demonstrate it again as shown in Fig. 5. In Fig. 5(a), when $L$ is larger than $0.44 \mu \mathrm{m}$, the thermomechanical states can reach critical condition and the sample fractures. Such size induced ductile to brittle transition, observed in the multi-scale simulations, is illustrated in Fig. 5(c). The critical thermomechanical states at fracture are shown in Fig. 5 (b), which are in agreement with those observed 
in full atomic simulations[30]. The close-up views of the deformation morphology near the shear band of the $0.88 \mu \mathrm{m}$ sample shown in the inset of Fig. 5 (c) demonstrate that the cavity forms in the shear band and soon propagates into a fatal crack. The crack propagates rapidly, resulting in intensive energy releasing and temperature rise, leaving two vein-like fracture surfaces with liquid-like droplets, in agreement with experimental observations[33,34] and the full atomic simulation[30].

\section{Simulation Results}

The multi-scale simulation method was first applied to Wahnstrom BLJ samples. The sample size $L$ studied ranges from $88 \mathrm{~nm}$ to $88 \mu \mathrm{m}$. The atomic region is a $21 \times$ $2.5 \times 92 \mathrm{~nm}^{3}$ slab, mimicking a portion near the shear band of the whole sample. The glassy slab is quenched from $2000 \mathrm{~K}$ to $10 \mathrm{~K}$ in $25 \mathrm{~ns}$. During the elastic loading with the strain rate of $0.02 \mathrm{~ns}^{-1}$, the shear band forms at around $5.5 \%$ strain in the atomic region. It should be noted that longer samples are reported to have lower yield stresses[4,16,30]. Here, we assumed all the samples yield at the same yield stress, thus slightly higher than their true yield points. As shown later, these samples do not cavitate with a $5.5 \%$ yield point, thus do not cavitate with their even lower true yield points. The strain rates for samples with different lengths are different: $2 \times 10^{-6}$, $2 \times 10^{-7}$, and $2 \times 10^{-8} \mathrm{ps}^{-1}$ for sample lengths of $0.88,8.8$ and 88 microns, respectively. In this way, the pulling end of the sample, assuming the other end of the sample is fixed, moving as a constant velocity.

The thermomechanical evolution of the shear band during uniaxial tension tests for samples with different lengths can be seen in Fig. 6(a). It should be noted that none of the samples exhibit cavitation during the multi-scale simulations, even for the longest sample. For long samples, according to Eq. (14), the tensile stress due to unloading via shear band gliding is very small. At the same time, the shear band heats up from the initial temperature $T_{i}(10 \mathrm{~K}$ here) due to plastic work. Thus, the shear band trajectory for long samples in the $\sigma_{z z}-T$ domain moves almost horizontally to the 
right. On the other hand, for short samples, the tensile stress drops quite readily. The shear band also heats up as the shear band glides. Due to the dropping tensile stress, once the acceleration (Eq. 11) turns negative and the gliding speed (Eq. 13) decreases, the shear band gradually stops gliding. Consequently, the temperature also drops due to heat dissipation to regions outside the shear band. Thus, the shear band trajectory for short samples in the $\sigma_{z z}-T$ domain moves rightward and downward, then leftward. These results are consistent with our previous reports using full atomic simulations [30].

Interestingly, the temperature increase of the shear band for the longest sample seems to slow down as seen in Fig. 6(a). This is due to the decreasing resistance (thus reduced heat generation rate) and the increasing heat conduction as the temperature increases (more discussion can be found in the next Section). Note the shear traction is mainly controlled by temperature but not shear strain rate.[21] Therefore, one can conjecture that there is a temperature upper limit for the shear band evolution, which corresponds to the balancing between heating from plasticity and cooling from conduction. Naturally, this high temperature limit (termed $T_{h}$ here) should be close to the glass transition temperature $T_{g}$. Similar consideration can also be found in the literature.[25] Based on this high temperature limit assumption and the negligible unloading (Eq. 14), for an infinitely long sample, the shear band trajectory in the $\sigma_{z z}-T$ domain is likely to be a straight line from $\left(T_{i}, \sigma_{z z}(0)\right)$ to $\left(T_{h}, \sigma_{z z}(0)\right)$, shown as in Fig. 6(a).

Since none of the samples cavitates in Fig. 6(a), the thermomechanical conditions for cavitation for BLJ system are unknown. As discussed in Ref. ([30]), the complete thermomechanical condition for cavitation inside a shear band is a concave envelope in the domain of the normal stress, shear stress and temperature of the shear band, i.e., $\sigma_{z z}-\sigma_{z x}-T$ domain. It has been observed for model metallic glasses under uniaxial tensile tests, the cavitation criterion can be simplified in the domain of $\sigma_{z z}-T$, due to the linear correlation between the critical tensile and shear stress. Here, 
the cavitation conditions for BLJ were examined in the $\sigma_{z z}-T$ domain by directly adjusting $\sigma_{z z}(0)$ and $\sigma_{x x}(0)$ the target stresses in the barostats. To do this, $\sigma_{z z}(0)$ and $\sigma_{x x}(0)$ were raised proportionally according to Eq. (1) and (3) by increasing $\sigma_{L}(0)$. It should be noted that, the high yield stress of $\sigma_{L}(0)$ might not be achieved under the normal uniaxial tension test protocol. Nonetheless, these tests can provide essentially a subset of the critical thermomechanical conditions for cavitation in the domain of $\sigma_{z z}-T$. The thermomechanical shear band trajectories for the longest sample using higher $\sigma_{z z}(0)$ were plotted in Fig. 6(a). With initial tensile stress of 2.5 GPa or higher, the longest sample cavitates. It should be stressed that the critical cavitation conditions (red crosses in Fig. 6a) have tensile stresses that are much higher than those accessible in the uniaxial tension tests.

\section{Discussions}

As shown in the preceding section, the shear bands in BLJ samples do not cavitate under uniaxial tension tests, since the shear band thermomechanical trajectory cannot reach the critical cavitation conditions. It has been shown before that shear bands in mBLJ samples do cavitate given that the sample is sufficiently long. Therefore, whether shear band cavitates under uniaxial tension seems to be strongly system dependent.

Let us first consider the general shear band trajectory in the $\sigma_{z z}-T$ domain as shown in Fig. 7(a). The normal stress of the shear band starts with $\sigma_{y} \sin ^{2} \theta$, with $\sigma_{y}$ the yield stress. As the shear band evolves, the tensile stress decreases and the temperature increases. The drop in tensile stress becomes less as the sample becomes longer. For infinitely long samples, the tensile stress can stay at $\sigma_{y} \sin ^{2} \theta$ without unloading. The shear band temperature increases from $T_{l}$, and saturates at $T_{h}$, the highest accessible temperature for shear band as it glides. This is consistent with the 
simulation results in Fig. 6(a). The rational for the existence of $T_{h}$ is that, as temperature increases, the viscosity of the shear band decreases rapidly. Therefore, the shear resistance, and the rate of heat generation reduce as the temperature goes up. However, the heat conduction increases with temperature and its gradient. If the shear band temperature goes above $T_{h}$, the heat production is lower than the heat conduction, which leads to temperature decreases, and vice versa. Therefore, $T_{h}$ is the result of balancing between heat production via plasticity and heat loss via conduction, which is likely to be close to the glass transition temperature (zero shear resistance and possible loss of localization).

Next, one can consider the critical cavitation conditions in the $\sigma_{z z}-T$ domain that separates the "no cavitation" region from the "cavitation" region. There are three apparent scenarios depending on the accessible thermomechanical states relative to the critical cavitation conditions. The first scenario is illustrated in Fig. 7(b), in which the initial thermomechanical state $\left(T_{l}, \sigma_{y} \sin ^{2} \theta\right)$ resides in the cavitation region. Once the shear band forms, it cavitates immediately regardless of the sample length. It should be noted that the sample may fracture (cleavage in the direction of maximum normal stress) prior to the formation of shear band. The second scenario (Fig. 7c) is such that the initial thermomechanical state is outside of the cavitation region, yet, accessible only for long samples. Thus, a size-dependency naturally emerges. mBLJ system appears to fit this scenario, so do typical experimental MG systems. In the third scenario, the accessible thermomechanical states of the shear band are outside of the cavitation region. Therefore, there is no shear band cavitation, thus no catastrophic tensile failure for the sample. BLJ system seems to fall into the third scenario. We termed the amorphous system belong to the above three scenarios as brittle-MGs, normal-MGs and cohesive-MGs.

It is of great interests to search for experimental cohesive-MG systems (similar to model BLJ system) that belong to the third scenario, which will avoid the highly undesirable tensile brittleness hindering the wider application of MG as structural materials. In addition to eradicate the tensile brittleness by reducing the length 
$[4,15,35,36]$, this study suggests that it is possible to eradicate the tensile brittleness of MG by tuning the chemistry. Although it is yet not clear precisely the chemical composition that leads to cohesive-MG systems, a useful hint can be obtained by the comparison between BLJ system (does not suffer from tensile brittleness, without covalent bonding) and mBLJ system (exhibits size-dependent tensile brittleness, with covalent bonding), that is, to reduce the covalent bonding.

\section{Conclusions}

A multi-scale simulation method was described here to model shear fracture in uniaxial tension tests on model MG, by combining an atomic region to describe shear band activities, and a continuum region to describe elasticity and heat transfer. This multi-scale method provides significant speed up over full-atomic simulations with comparable results, and allows investigations on samples as long as 88 microns. As the sample length increases, the elastic unload in tensile stress of the shear band diminishes during shear band gliding. On the other hand, the temperature of the shear band increases during shear band development and appears to be limited by an upper bound due to reduced shear resistance and increased heat conduction. These observations suggest that the model binary Lennard-Jones model metallic glass does not suffer from shear band cavitation, even with infinite length. Lastly, based on the initial tensile stress of the shear band, the upper bound for shear band temperature, as well as the critical thermomechanical conditions for shear band cavitation, we proposed three scenarios to categorize the amorphous system: brittle regardless of length (brittle-MGs); ductile to brittle as sample length increases (normal-MGs); ductile regardless of length (cohesive-MGs). An experimental cohesive-MG system with minimal covalent bonding will exhibit intrinsic tensile plasticity even for macroscopic samples. 


\section{Acknowledgements}

We thank the support from the National Science Foundation-United States under grant DMR-1207439, XSEDE under award TG-DMR130089, and the Center for Computational Innovations at Rensselaer Polytechnic Institute. 


\section{Reference}

[1] Demetriou, M. D. et al. A damage-tolerant glass. Nature Materials 10, 123-128 (2011).

[2] Schroers, J. \& Johnson, W. L. Ductile bulk metallic glass. Phys Rev Lett 93, 255506 (2004).

[3] Das, J. et al. 'Work-Hardenable' Ductile Bulk Metallic Glass. Physical Review Letters 94, 205501 (2005).

[4] Jang, D. \& Greer, J. R. Transition from a strong-yet-brittle to a stronger-and-ductile state by size reduction of metallic glasses. Nature Materials 9, 215-219 (2010).

[5] Kumar, G., Tang, H. X. \& Schroers, J. Nanomoulding with amorphous metals. Nature 457, 868-872 (2009).

[6] Johnson, W. L. et al. Beating Crystallization in Glass-Forming Metals by Millisecond Heating and Processing. Science 332, 828-833 (2011).

[7] Chen, M., Inoue, A., Zhang, W. \& Sakurai, T. Extraordinary Plasticity of Ductile Bulk Metallic Glasses. Physical Review Letters 96, 245502 (2006).

[8] Liu, Y. H. et al. Super Plastic Bulk Metallic Glasses at Room Temperature. Science 315, 1385-1388 (2007).

[9] Hays, C., Kim, C. \& Johnson, W. Microstructure Controlled Shear Band Pattern Formation and Enhanced Plasticity of Bulk Metallic Glasses Containing in situ Formed Ductile Phase Dendrite Dispersions. Physical Review Letters 84, $2901-2904$ (2000). 
[10] Hofmann, D. C. et al. Designing metallic glass matrix composites with high toughness and tensile ductility. Nature 451, 1085-1089 (2008).

[11] Sarac, B. \& Schroers, J. Designing tensile ductility in metallic glasses. Nature Communications 4, (2013).

[12] Wang, Z. T., Pan, J., Li, Y. \& Schuh, C. A. Densification and Strain Hardening of a Metallic Glass under Tension at Room Temperature. Physical Review Letters 111, (2013).

[13] Qu, R. T., Calin, M., Eckert, J. \& Zhang, Z. F. Metallic glasses: Notch-insensitive materials. Scripta Materialia 66, 733-736 (2012).

[14] Wang, Q. et al. Superior Tensile Ductility in Bulk Metallic Glass with Gradient Amorphous Structure. Scientific Reports 4, (2014).

[15] Guo, H. et al. Tensile ductility and necking of metallic glass. Nature Materials 6, $735-739$ (2007).

[16] Wang, C.-C. et al. Sample size matters for Al88Fe7Gd5 metallic glass: Smaller is stronger. Acta Materialia 60, 5370-5379 (2012).

[17] Yi, J., Wang, W. H. \& Lewandowski, J. J. Sample size and preparation effects on the tensile ductility of Pd-based metallic glass nanowires. Acta Materialia 87, $1-7$ (2015).

[18] Greer, A. L., Cheng, Y. Q. \& Ma, E. Shear bands in metallic glasses. Materials Science and Engineering: R: Reports 74, 71-132 (2013).

[19] Greer, J. R. \& De Hosson, J. T. M. Plasticity in small-sized metallic systems: Intrinsic versus extrinsic size effect. Progress in Materials Science 56, 654-724 
(2011).

[20] Kuzmin, O. V., Pei, Y. T., Chen, C. Q. \& De Hosson, J. T. M. Intrinsic and extrinsic size effects in the deformation of metallic glass nanopillars. Acta Materialia 60, 889-898 (2012).

[21] Cheng, Y., Han, Z., Li, Y. \& Ma, E. Cold versus hot shear banding in bulk metallic glass. Physical Review B 80, 134115 (2009).

[22] Wu, F. F., Zhang, Z. F. \& Mao, S. X. Size-dependent shear fracture and global tensile plasticity of metallic glasses. Acta Materialia 57, 257-266 (2009).

[23] Yang, Y. \& Liu, C. T. Size effect on stability of shear-band propagation in bulk metallic glasses: an overview. Journal of Materials Science 47, 55-67 (2011).

[24] Spaepen, F. Metallic glasses: Must shear bands be hot? Nature Materials 5, 7-8 (2006).

[25] Shimizu, F., Ogata, S. \& Li, J. Yield point of metallic glass. Acta Materialia 54, 4293-4298 (2006).

[26] Shimizu, F., Ogata, S. \& Li, J. Theory of shear banding in metallic glasses and molecular dynamics calculations. Materials transactions 48, 2923-2927 (2007).

[27] Guan, P., Lu, S., Spector, M. J. B., Valavala, P. K. \& Falk, M. L. Cavitation in Amorphous Solids. Physical Review Letters 110, 185502 (2013).

[28] Murali, P., Narasimhan, R., Guo, T. F., Zhang, Y. W. \& Gao, H. J. Shear bands mediate cavitation in brittle metallic glasses. Scripta Materialia 68, 567-570 (2013).

[29] Murali, P. et al. Atomic Scale Fluctuations Govern Brittle Fracture and 
Cavitation Behavior in Metallic Glasses. Physical Review Letters 107, 215501 (2011).

[30] Luo, J. \& Shi, Y. Tensile fracture of metallic glasses via shear band cavitation. Acta Materialia 82, 483-490 (2015).

[31] Shi, Y. \& Falk, M. Strain Localization and Percolation of Stable Structure in Amorphous Solids. Physical Review Letters 95, 095502 (2005).

[32] Müller-Plathe, F. A simple nonequilibrium molecular dynamics method for calculating the thermal conductivity. The Journal of Chemical Physics 106, 6082 (1997).

[33] Qu, R. T., Stoica, M., Eckert, J. \& Zhang, Z. F. Tensile fracture morphologies of bulk metallic glass. Journal of Applied Physics 108, 063509 (2010).

[34] Zhang, Z. ., Eckert, J. \& Schultz, L. Difference in compressive and tensile fracture mechanisms of $\mathrm{Zr} 59 \mathrm{Cu} 20 \mathrm{Al10Ni8Ti3}$ bulk metallic glass. Acta Materialia 51, 1167-1179(2003).

[35] Luo, J. H., Wu, F. F., Huang, J. Y., Wang, J. Q. \& Mao, S. X. Superelongation and Atomic Chain Formation in Nanosized Metallic Glass. Physical Review Letters 104, (2010).

[36] Tian, L., Shan, Z.-W. \& Ma, E. Ductile necking behavior of nanoscale metallic glasses under uniaxial tension at room temperature. Acta Materialia 61, 4823-4830 (2013). 


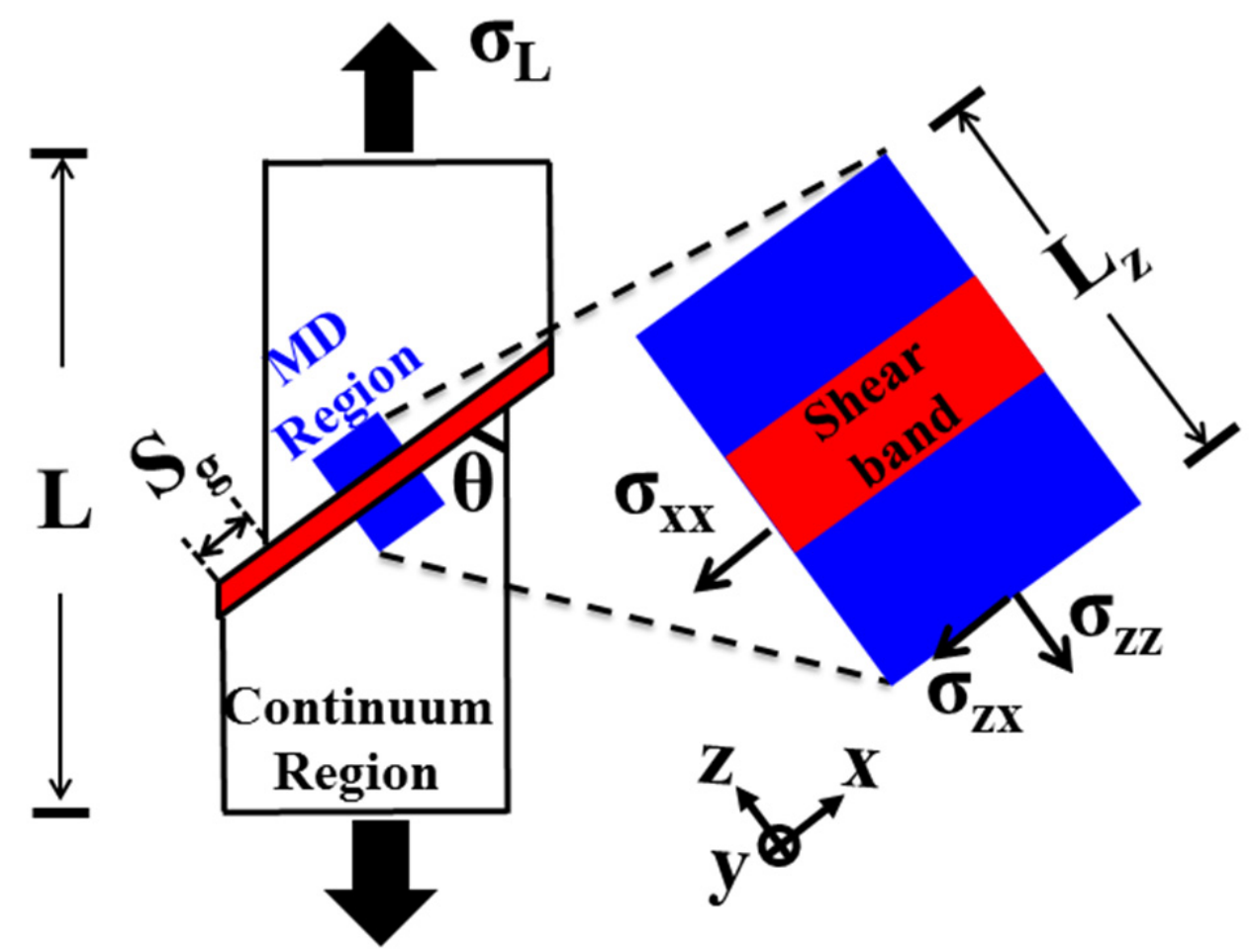

Figure 1. Illustration of the multi-scale simulation strategy on a uniaxial tensile test of a metallic glass sample, which exhibits a single dominant shear band (colored red). The MD region describes part of the shear band and its vicinity (zoomed-in view on the right), while the rest of the sample is treated at the continuum level. 


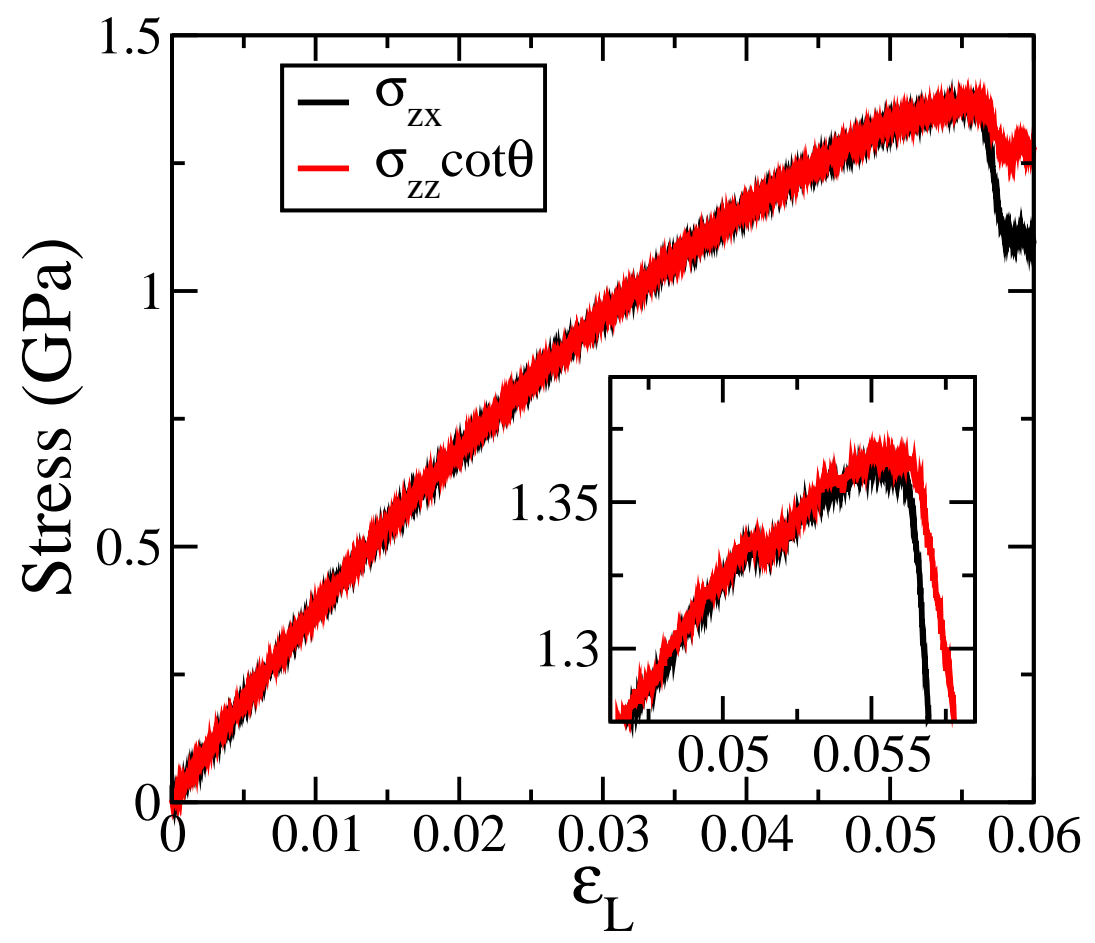

Figure 2. The driving shear stress $\sigma_{z z} \cot \theta$ and the resisting shear stress $\sigma_{z x}$ as a function of the tensile strain. The elastic deformation ends approximately around $5.5 \%$ strain, at which the driving shear stress becomes higher than the resisting shear stress. The inset shows a zoomed-in view of the same plot. 


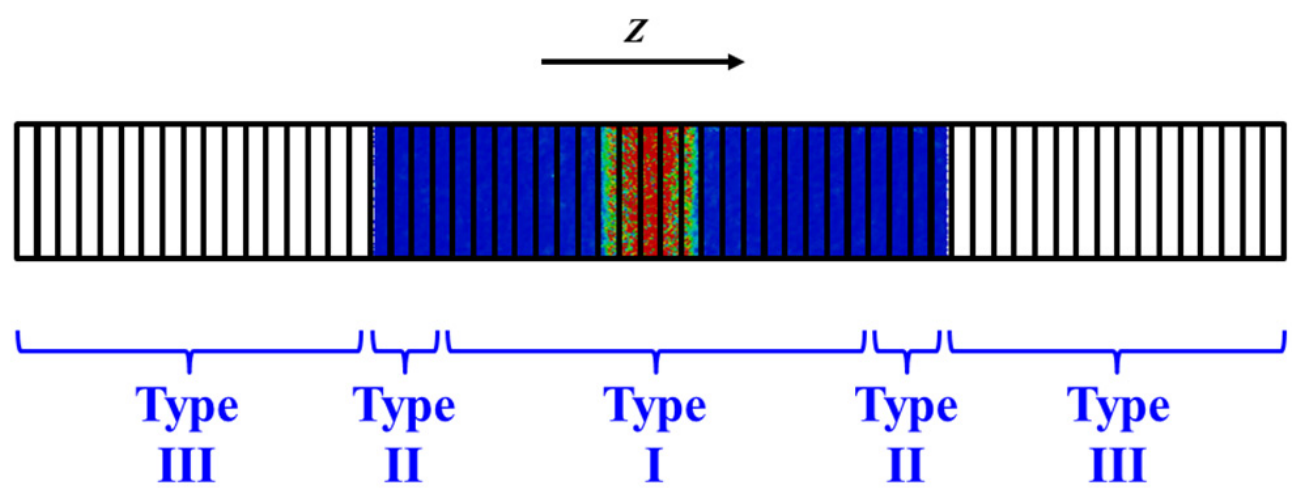

Figure 3. Illustration of the thermal coupling between the continuum region and the MD region. The continuum, course-grained description spans over the entire sample along the z-direction (normal to the shear band, as in Fig. 1). The MD region spans over both Type I and II regions. The detailed coupling can be found in the main text. 


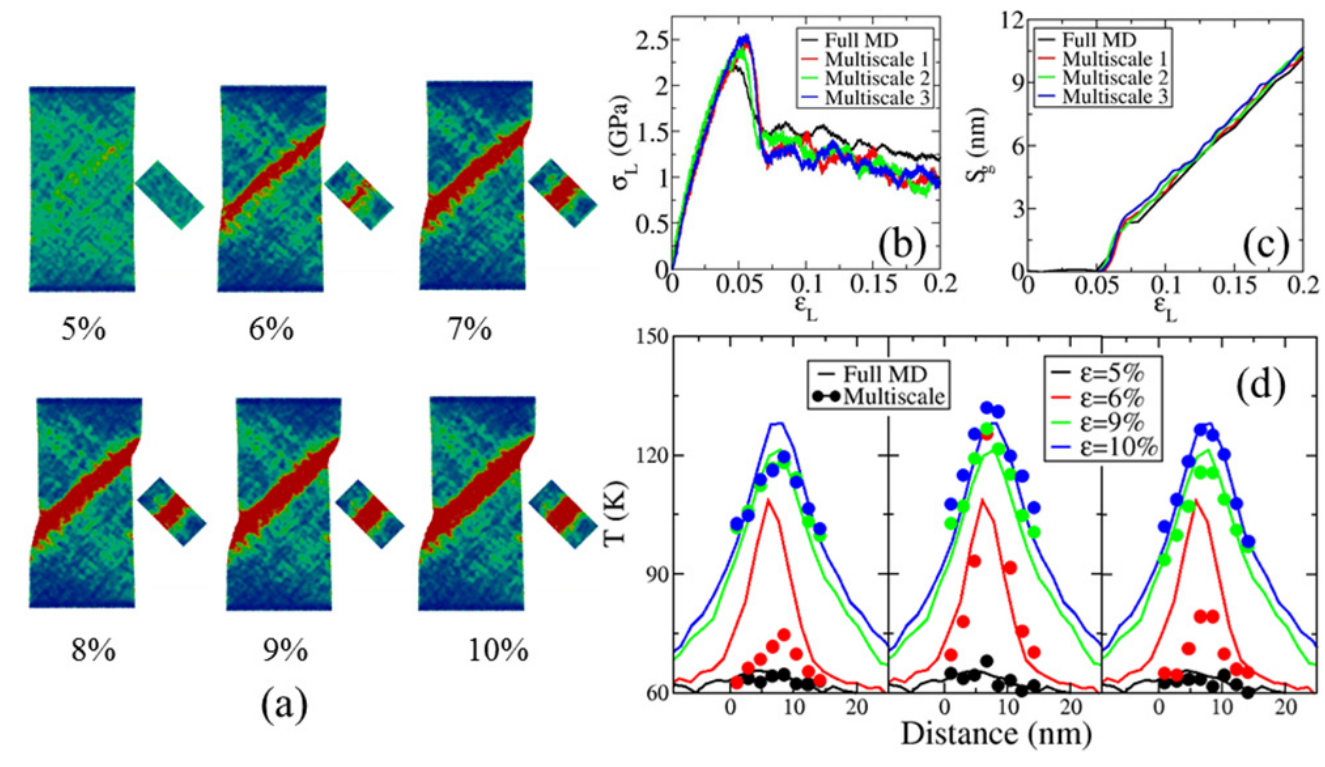

Figure 4. Comparison of the results of full MD simulation and the multi-scale simulations: (a) deformation morphologies (full MD simulations on the left and MD region of the multi-scale simulation on the right, tilted) at different tensile strains (blue denotes zero strain to red denotes local strains from $0 \%$ to $20 \%$ or more); (b) stress-strain curves; (c) gliding distance; (d) temperature distributions perpendicular to the shear band. Note that there are three independent multi-scale simulations shown in panes (b-d) to show the sample-to-sample variations. 

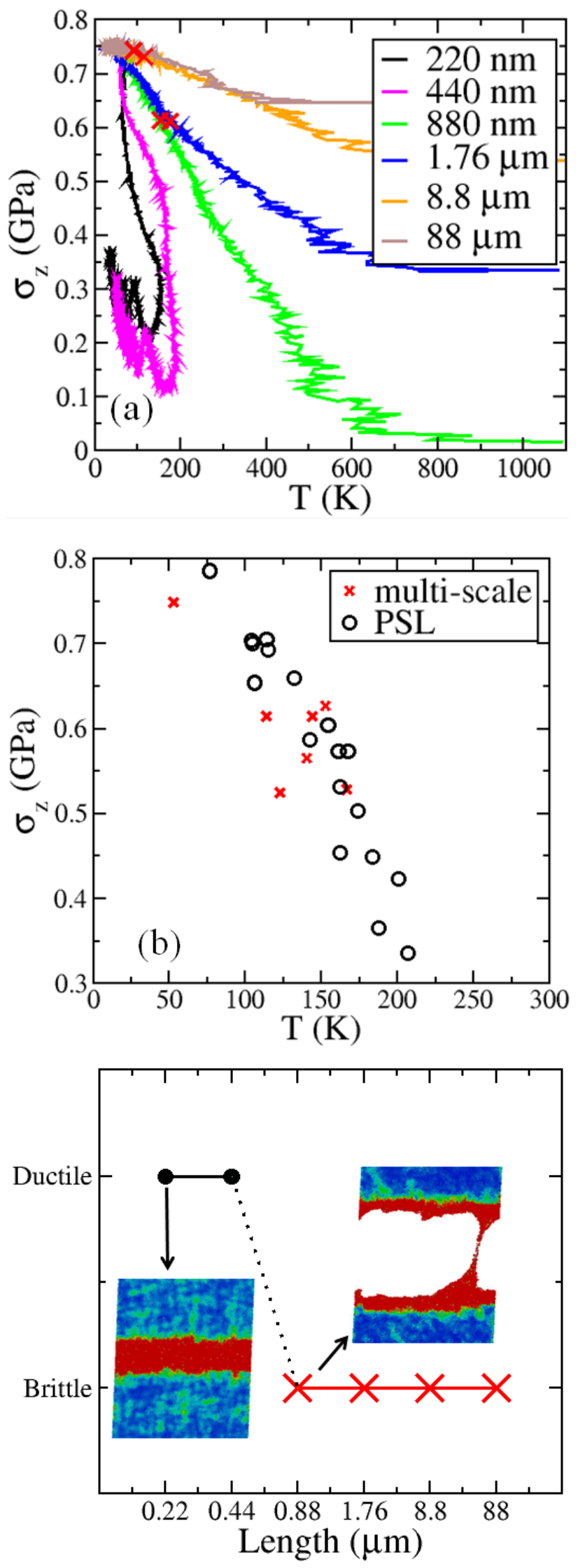
Figure 5. (a) Trajectories of the shear band in the domain of $\sigma_{z}$ and $T$ for the modified BLJ samples with sizes from $220 \mathrm{~nm}$ to $88 \mu \mathrm{m}$. A ductile to brittle transition is seen when the size increases beyond $440 \mathrm{~nm}$, which is illustrated in (c). Red crosses indicate the cavitation states. (b) Cavitation states collected from the multi-scale simulations and full atomic simulations using perturbative static load (PSL) [30]. (c) Multiscale simulations show the ductile to brittle transition induced by increasing the sample length. The insets show the representative deformation morphologies of the atomic regions of the multiscale simulations corresponding to ductile (no cavitation) and brittle (cavitation, and subsequent fracture) behaviors. The atoms are colored according to the local shear strain (blue denotes zero strain, red denotes $20 \%$ or more). 

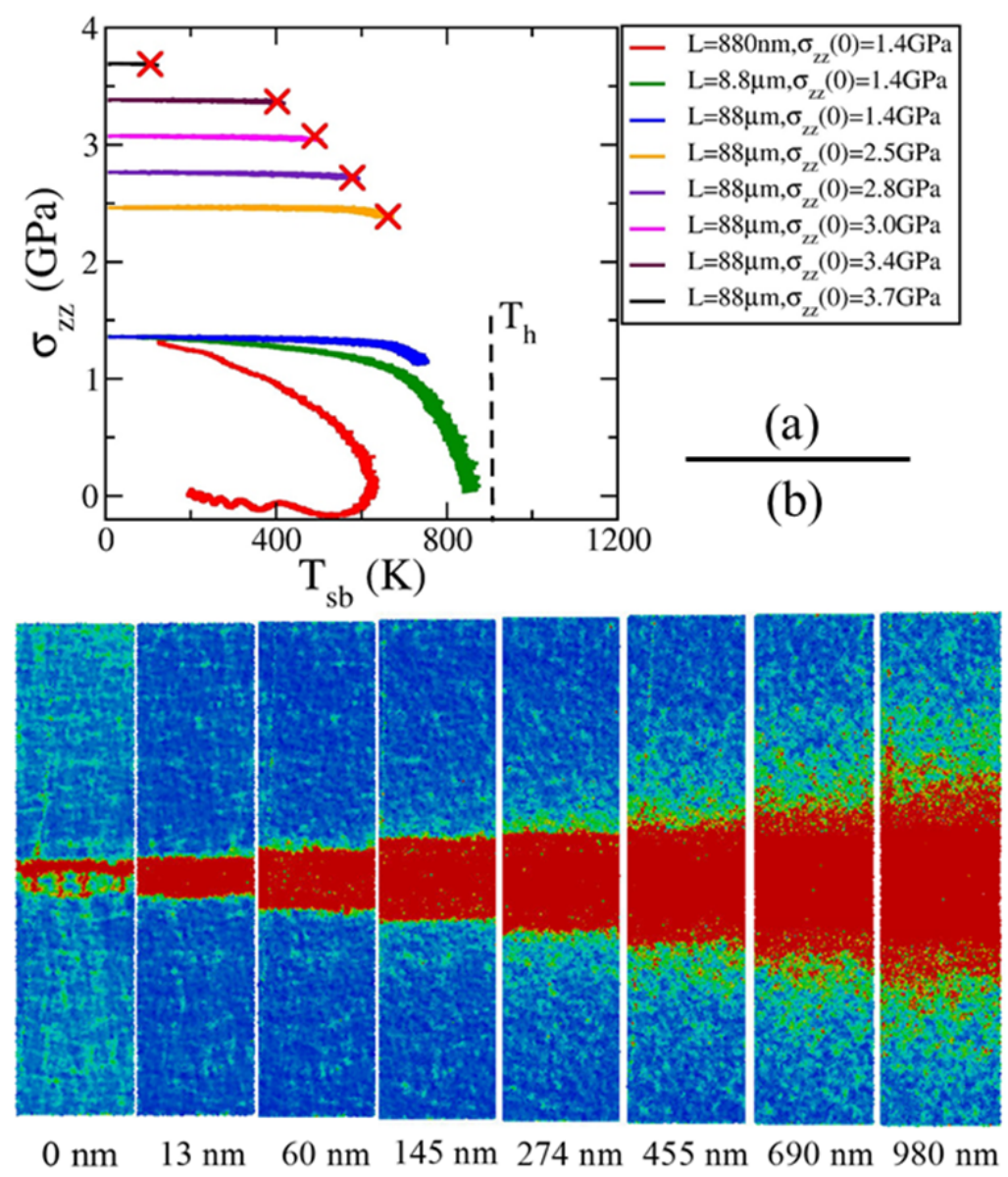

Figure 6. (a) The thermomechanical evolution of the shear band in the domain of $\sigma_{z z}-T$ in samples with different lengths $(L)$ and different initial tensile stress at the beginning of plastic deformation $\left(\sigma_{z z}(0)\right)$. The original $\sigma_{z z}(0)$ for uniaxial tension tests with a yield strain around $5 \%$ is about $1.4 \mathrm{GPa}$. We artificially elevated $\sigma_{z z}(0)$ in order to map out the cavitation condition. The red crosses indicate cavitation. $T_{h}$ denotes the upper limit of the shear band temperature. (b) The deformation morphologies at listed gliding distances $\boldsymbol{S}_{\boldsymbol{g}}$ are shown for the sample with L=88 $\mu \mathrm{m}$. The atoms are colored according to the local shear strain (blue denotes zero strain, red denotes $20 \%$ or more). 

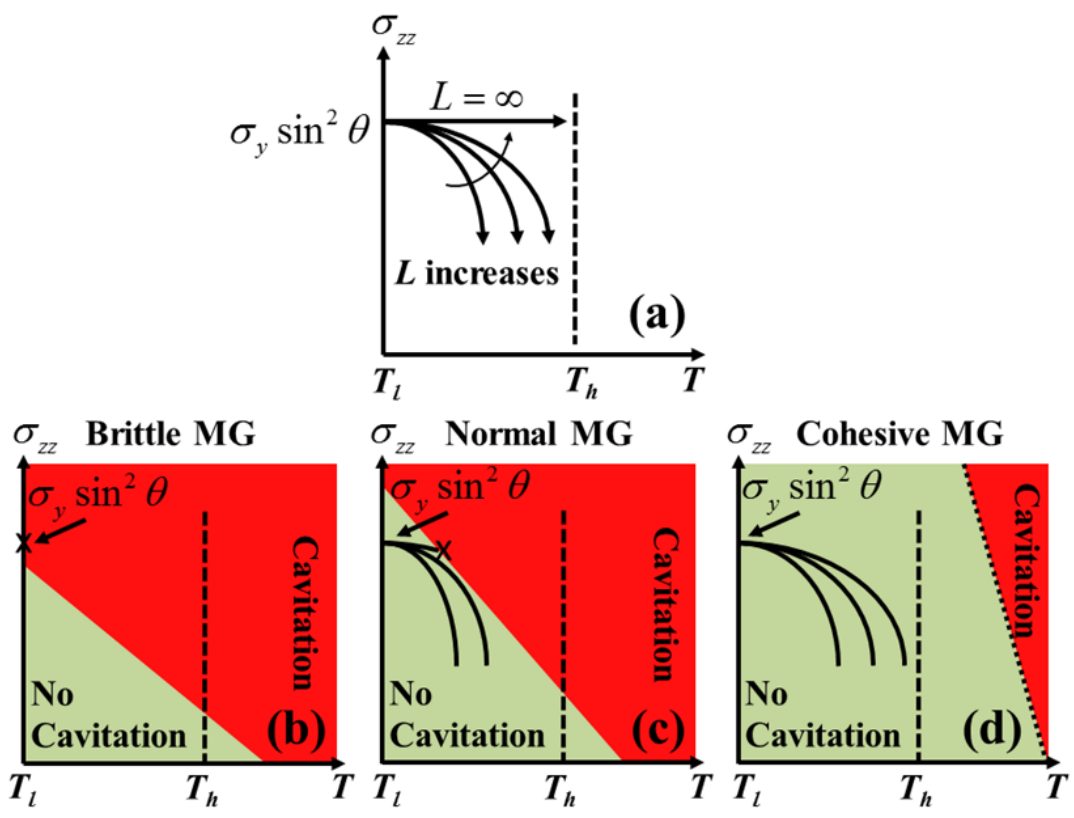

Figure 7. (a) Illustration of the shear band thermomechanical trajectory (in the domain of $\sigma_{z z}-T$ ) under uniaxial tension for different sample length. For infinitely long sample, the trajectory becomes a straight line to the right, ending at $T_{h}$ (the upper limit of the shear band temperature). $T_{l}$ is the initial temperature of the glass before mechanical tests. (b-d) Three scenarios for metallic glass systems in terms of tensile ductility: brittle-MG, normal-MG and cohesive-MG. $\sigma_{y} \sin ^{2} \theta$ indicates the initial tensile stress of the shear band just after yielding. The red region is the cavitation zone. Brittle fractures are indicated by a black cross. (b) For brittle-MG systems, the initial thermomechanical state of the shear band $\left(\sigma_{y} \sin ^{2} \theta, T_{l}\right)$ is in the cavitation zone, thus exhibit size-independent brittle fracture. (c) For normal-MG systems, the initial thermomechanical state of the shear band is not in the cavitation zone. However, for long samples, the thermomechanical trajectory can reach the cavitation zone, leading to size-dependent tensile brittleness. (d) For cohesive-MG systems, the entire thermomechanical trajectory of the shear band locates inside the no-cavitation zone, thus exhibit size-independent tensile ductility. 


\title{
A model metallic glass exhibits size-independent tensile ductility
}

Jian Luo, Pawel Keblinski, and Yunfeng Shi

Department of Materials Science and Engineering,

Rensselaer Polytechnic Institute, Troy, NY 12180

\begin{abstract}
Metallic glasses (MGs) usually suffer brittle fracture under uniaxial tension due to size-dependent shear band cavitation. Here we developed a concurrent multi-scale simulation method to describe the uniaxial tension of a binary Lennard-Jones (BLJ) model glass up to 88 microns in length.
\end{abstract}

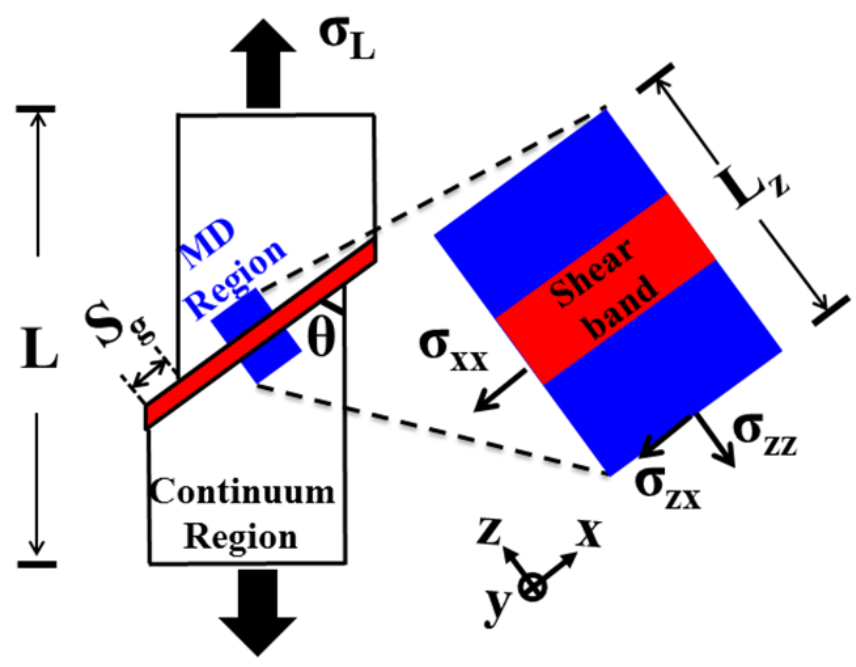

No cavitation or brittle fracture was found even for the longest BLJ sample. As the length increases, the shear band temperature increases, then saturates, while the elastic unloading from shear-off diminishes. 

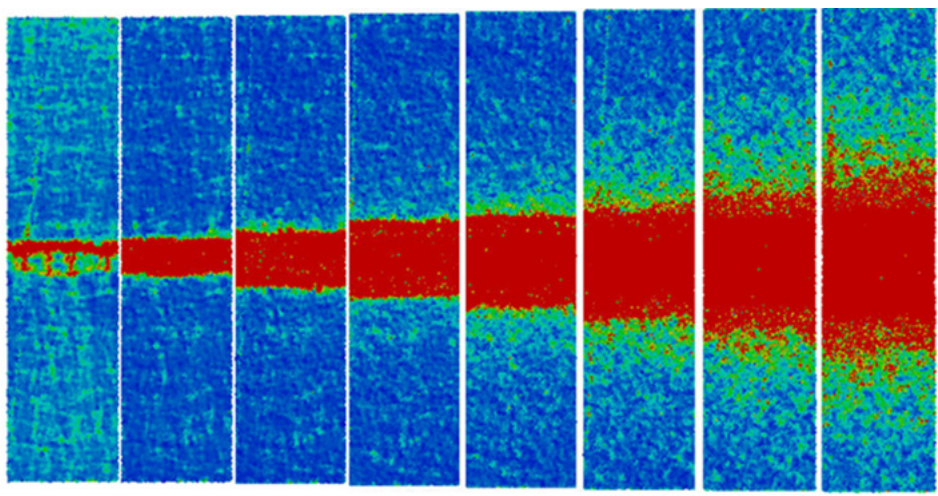

We conjecture that the shear band of a BLJ sample, even with a macroscopic length, cannot reach the herein estimated critical cavitation conditions. Thus, BLJ samples appear to be free of size-induced tensile brittleness.
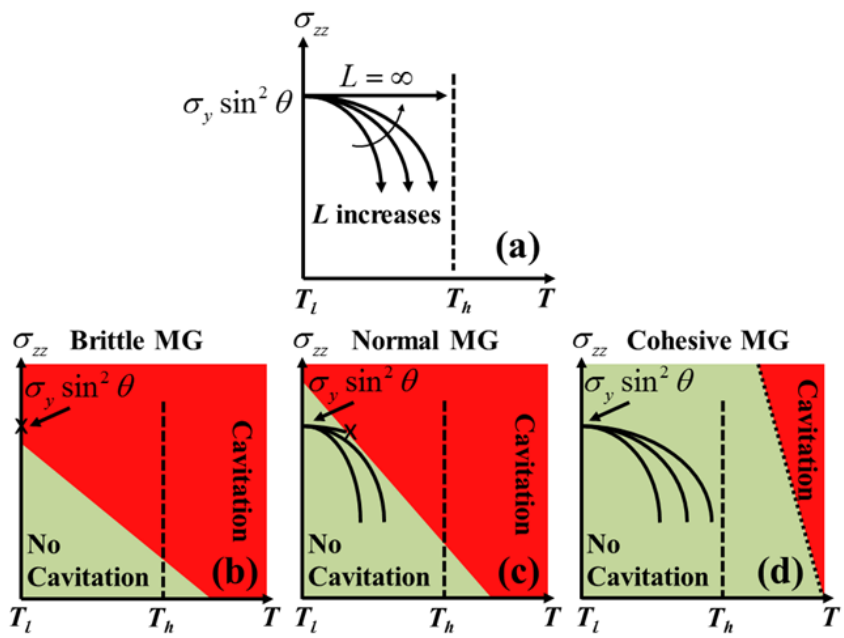

Based on the shear band evolution and the critical cavitation conditions, we propose three classes of MGs in terms of tensile ductility: brittle-MGs (brittle for all lengths), normal-MGs (ductile for short samples, brittle for long samples), cohesive-MGs (ductile for all lengths).

Our simulation results illustrate limitations of existing molecular models, and suggest that certain experimental metallic glasses may be free of size-induced tensile brittleness. 